Notice critique à propos du document « Le Bonheur, émané de la Liberté ». Lettre [de Robespierre ?] à un destinataire inconnu, non datée [1792]

\title{
Marco Marin
}

\section{OpenEdition}

\section{Journals}

Édition électronique

URL : https://journals.openedition.org/ahrf/13511

DOI : 10.4000/ahrf.13511

ISSN : 1952-403X

Éditeur :

Armand Colin, Société des études robespierristes

Édition imprimée

Date de publication : 1 juin 2015

Pagination : 125-132

ISBN : 9782200929718

ISSN : 0003-4436

Référence électronique

Marco Marin, « Notice critique à propos du document « Le Bonheur, émané de la Liberté ». Lettre [de Robespierre ?] à un destinataire inconnu, non datée [1792] », Annales historiques de la Révolution française [En ligne], 380 | juin 2015, mis en ligne le 01 juin 2018, consulté le 24 avril 2022. URL : http:// journals.openedition.org/ahrf/13511; DOI : https://doi.org/10.4000/ahrf.13511 


\title{
NOTICE CRITIQUE À PROPOS DU DOCUMENT «LE BONHEUR, ÉMANÉ DE LA LIBERTÉ». LETTRE [DE ROBESPIERRE ?] À UN DESTINATAIRE INCONNU, NON DATÉE [1792]
}

\author{
Marco MARIN
}

Dans son premier numéro de $2013^{2}$, les Annales Historiques de la Révolution Française se sont fait l'écho de l'acquisition, en novembre 2011, d'un ensemble de 126 pages manuscrites « de » Maximilien Robespierre ${ }^{3}$, avec le soutien d'une souscription publique lancée par la SER. Ces documents sont désormais conservés aux Archives nationales (AN).

Ce précieux ensemble de textes avait été mis en vente par des descendants du conventionnel Philippe Le Bas (1764-1794), compagnon de l'Incorruptible, né à Frévent (Pas-de-Calais), qui se donna la mort à l'occasion de l'attaque de l'Hôtel de Ville par les troupes de la Convention dans la nuit entre le 9 et le 10 Thermidor an II. Afin de compléter la présentation, ainsi qu'une première analyse de l'ensemble documentaire par Annie Geffroy ${ }^{4}$, cette note souhaite se pencher sur l'un des deux textes inédits du dossier, présenté comme «Le Bonheur, émané de la Liberté »/lettre à un destinataire inconnu, non datée [1792] ». Il s'agit particulièrement de s'interroger sur la graphie, l'auteur et le destinataire -

(1) Lettre conservée aux Archives nationales, 683 AP/1 (dossier 2).

(2) Annales historiques de la Révolution française $(A H R F), \mathrm{n}^{\circ} 371$, janvier-mars 2013. Cf. en particulier les articles de Serge ABERDAM, Cyril TRIOLAIRE, «La souscription nationale pour sauvegarder les manuscrits de Robespierre : introspection historique d'une initiative citoyenne et militante », p. 9-39 ; Annie GEFFROY, « Les manuscrits de Robespierre », p. 39-54.

(3) Les guillemets sont utilisés par Annie GEFFROY, « Les manuscrits de Robespierre », AHRF, $\mathrm{n}^{\circ} 371$, op. cit. Il faut noter que notre lecture du manuscrit présente certaines différences par rapport à la transcription proposée dans le $n^{\circ} 371$ des $A H R F$. Dans le recto, ligne 1 , on retrouve «suffisait » à la place de « suffisoit »; ligne 4, après « celui » il n'y a pas de virgule ; ligne 20 « nature entiere » (raturé) paraît en minuscule ; verso, ligne 22 « cette maxime » nous paraît plutôt être « celle maxime » ; ligne 30 le dernier mot n'est pas - selon nous - « commée » mais « comment ».

(4) Annie GEFFROY, « Les manuscrits de Robespierre », $A H R F, \mathrm{n}^{\circ} 371$, op. cit. 
réel ou hypothétique - du document désormais conservé en AN, 683 AP/1 (dossier 2).

Comme nous pouvons le constater par les versions numérisées des documents présents sur la base de données ARCHIM, la graphie de « Bonheur, émané de la Liberté » diffère sensiblement de celle de la plupart des autres feuilles achetées en $2011^{5}$. Le « dossier 2 » a en effet été rédigé - comme l'a récemment remarqué Hervé Leuwers ${ }^{6}$ - avec une graphie plutôt ronde, ample et lisible, différente de celle de Robespierre ; tandis que la plupart des autres ${ }^{7}$ documents ont été rédigés avec une écriture serrée et fine, presque nerveuse.

Puisqu'aucun des documents récemment acquis ne présente la signature de l'auteur, il a été d'abord nécessaire de comparer les diverses graphies avec certains manuscrits autographes de Robespierre, conservés aux Archives nationales. Grâce au troisième tome des Euvres de Robespierre ${ }^{8}$, nous avons ainsi pu repérer les originaux de certaines lettres écrites et signées par l'Incorruptible qui remontent à l'époque de sa participation au Comité de salut public (cartons des AN AF/II/57, AF/II/58, AF/II/244, $\mathrm{AF} / \mathrm{II} / 301$ ). La graphie de ces lettres ainsi que la signature correspondent parfaitement à la graphie de la plupart des fragments récemment achetés, c'est-à-dire au trait distinctif de la main de Robespierre, et diffère tout à fait de la calligraphie présente dans le « dossier $2 »$.

Or, si l'on élimine l'hypothèse - qui nous paraît impossible - selon laquelle Robespierre aurait deux façons d'écrire très différentes entre elles, qui a donc pu rédiger le « dossier $2 »$ ?

Afin de trouver des indices, nous nous sommes appuyés sur l'historiographie érudite jadis dédiée à l'Incorruptible. Hamel, Stéfane-Pol (pseud. de Paul Coutant), Fleischmann, Grasilier ${ }^{9}$, indiquent tous, à partir

(5) Seul le fragment du dernier discours Contre les factions nouvelles et les députés corrompus, (8 thermidor an II, 26 juillet 1794), AN, 683 AP/1 (dossier 12), qui est indiqué comme une copie de l'original, paraît être rédigé par la même main de l'auteur du « Bonheur émané de la Liberté ».

(6) Hervé Leuwers, avec la responsable de la section des « Archives privées » des Archives nationales, Isabelle Aristide, a tenu le 25 juin 2013 une conférence intitulée « Des manuscrits de Robespierre à la loupe $(683 \mathrm{AP})$ » dans le cadre du cycle de rencontres des « Trésors du patrimoine écrit », organisé par l'Institut national du patrimoine.

(7) Cf., par exemple, le fragment du Discours sur la guerre, aux Jacobins du 25 janvier 1792 (AN, $683 \mathrm{AP} / 1$, dossier 3).

(8) Maximilien RobesPIERRE, Euvres (1910-2007), 11 vol., Paris, SER, rééd. 2011.

(9) Cf. Ernest HAMEL, Histoire de Robespierre d'après des papiers de famille les sources originales et des documents entièrement inédits, 3 tomes, Paris, chez l'auteur, 1867, en particulier t. 1, chap. XXIX et t. 3, chap. XXIX, p. 281 et sq; idem, La maison de Robespierre, Paris, A. Charles, 1895, en particulier p. 11 ; STÉFANE-POL, Autour de Robespierre : Le conventionnel Le Bas, d'après des documents inédits et les mémoires de sa veuve, Paris, Flammarion, 1901, p. 83 ; Hector FLEISCHMANN, 
de documents et de témoignages de l'époque, que Robespierre, pendant une durée non précisée, put bénéficier de la collaboration de Simon Duplay (1774-1827) en qualité de secrétaire. Peut-on alors attribuer à ce personnage la responsabilité de l'écriture de la lettre à un destinataire inconnu récemment retrouvée?

Un premier indice peut être trouvé dans la vie même de Simon Duplay qui, à la fin de 1792, habita avec Robespierre. Une fois devenu orphelin, il fut accueilli, avec son frère Jacques, par son oncle paternel, le menuisier Maurice Duplay ${ }^{10}$, qui hébergea également Maximilien après le 17 juillet 1791 - et après le 9 août 1791 selon Mathiez ${ }^{11}$. Il est également important de rappeler que Simon Duplay, après son engagement volontaire au $1^{\text {er }}$ novembre 1791 et à la suite de la perte d'une jambe ${ }^{12}$ dans la célèbre bataille de Valmy du 20 septembre 1792, travailla pour l'administration républicaine : à partir du $1^{\mathrm{er}}$ mai 1793 comme « expéditionnaire aux bureaux de la gendarmerie », puis, à partir du 6 prairial an II (15 mai 1794), en tant qu' « adjoint » d'Augustin Le Jeune auprès du Bureau de Police, créé - grâce à l'insistance de Robespierre et Saint-Just - au début floréal de l'an $\mathrm{II}^{13}$. Le fait que, au cours de cette deuxième tâche, Simon put approfondir son rapport personnel et professionnel avec l'Incorruptible, constitue une donnée historiquement démontrée, puisque les deux travaillèrent côte à côte. Mais fut-il aussi son secrétaire ?

Même s'il existe une littérature importante relative aux secrétaires de Robespierre, l'attribution à Maximilien d'un ou de plusieurs d'entre

Robespierre et les femmes, d'après des documents nouveaux et des pièces inédites..., Paris, A. Michel, 1909 ; Léonce GRASILIER, Un secrétaire de Robespierre. Simon Duplay (1774-1827) et son Mémoire sur les sociétés secrètes et les conspirations sous la Restauration, Paris, 1913 (cf. aussi par rapport au texte de Grasilier le compte rendu de Mathiez dans les Annales révolutionnaires de 1913, p. 418-419). J'ai pu consulter seulement une très mauvaise édition du texte de Fleischmann, tirée du texte numérique (Memphis, General Books LLC, 2012). Par rapport à Simon Duplay voir en particulier p. 26, troisième colonne.

(10) Pour une courte notice biographique sur Maurice Duplay voir Ernest HAMEL, Histoire de Robespierre, op. cit., t. 3, chap. XXIX.

(11) Mathiez utilise la déposition de Robespierre comme témoin du massacre du Champ de Mars (9 août 1791) pour affirmer que Robespierre rentre chez les Duplay uniquement dans la deuxième moitié du mois d'août. Pour l'occasion, en listant ses coordonnées, Robespierre indique comme son adresse le 8, rue de Saintonge, c'est-à-dire l'adresse où il habita pendant la période de la Constituante. Cependant, en août 1791, Robespierre habitait probablement déjà chez des Duplay, dans l'attente de prendre une décision définitive. Cf. Albert MATHIEZ, « À quelle date Robespierre s'est-il installé chez Duplay ? », Annales révolutionnaires, janv.-déc. 1910, p. 106-107.

(12) La question de savoir quelle jambe a été perdue par Simon est assez drôle. Selon Fleischmann (ed. 2012, p. 26) et Stéfane-Pol (p. 83) il s'agit de la jambe gauche, selon Grasilier (p. 7) plutôt de la droite. Les documents conservés aux AN, dans F/7/4697, qui s'arrêtent plusieurs fois sur la jambe amputée, n'arrivent pourtant pas à éliminer tout doute.

(13) Arne ORDING, Le Bureau de police du Comité de Salut public. Étude sur la Terreur, Oslo, i kommisjon hos J. Dybwad, 1930. 
eux demeure toujours incertaine ${ }^{14}$. Par exemple, celui qui est considéré de fait comme le chef de Simon Duplay, à savoir Le Jeune, est défini au moins dans une occasion en tant que secrétaire de Maximilien ${ }^{15}$. Quoi qu'il en soit, Simon Duplay fut vraisemblablement un collaborateur proche de Robespierre, tout au moins à certaines périodes bien délimitées.

Parmi les historiens qui se sont occupés de ces questions, seul Arne Ording $(1930)^{16}$, suivant une affirmation de Simon Duplay, nie qu'il ait accompli les fonctions de secrétaire de Robespierre. Cependant, l'interrogatoire de Simon sur lequel Ording se base est une source très contestable. En effet, lorsqu'il fut écouté par l'autorité judiciaire, Simon risquait la guillotine pour complicité avec la « conjuration inachevée » du triumvirat de Robespierre, Saint-Just et Couthon. Il est alors logique de le voir nier tout rapport de proximité avec Maximilien :

« Citoyens Representans, j'ai été arrété le 12 thermidor/par ordre du comité de Sureté générale. et l'ordre/portait : le Secretaire de Robespierre. je ne sais qui/a pu me donner un titre que je n'ai jamais eu. et/vous n'ignorés-pas vous mêmes citoyens Representans que/Robespierre n'avoit point de Sécrétaire, qu'il ne/donnait pas même ses discours à copier [...] j'ai eu le malheur detre $[s i c]$ accueilli/à mon retour de l'armée par mon oncle/ches [sic] qui logeait Robespierre. il m'a trompé/comme tant d'autres, voilà tous mes crimes $»^{17}$.

Ainsi, nous avançons l'hypothèse selon laquelle Simon Duplay a été le secrétaire - ou du moins le copiste - de Robespierre entre les derniers mois de 1792 et le $1^{\text {er }}$ mai 1793 et ensuite entre le 6 prairial (15 mai 1794) et le 9 thermidor an II (27 juillet 1794). Nous avons alors sondé les archives à la recherche d'un document rédigé et personnellement signé par Simon pour pouvoir comparer sa graphie avec celle du « dossier $2 »$.

(14) À propos des secrétaires de Robespierre cf. note 9 supra. Outre Simon et Le Jeune, on a également parlé de secrétaires de Robespierre qui auraient été François Bègue et Pierre Villiers. Cf. Jean PALOU, «Un prétendu secrétaire de Robespierre : Bègue dit Magloire (1750?-1837) », $A H R F, 1947, n^{\circ} 3$, p. 257-261 ; Roger BARMY, «Aux origines de la légende anti-robespierriste : Pierre Villiers et Robespierre ", dans Actes du colloque Robespierre. XII ${ }^{e}$ Congrès international des Sciences historiques (Vienne, 3 sept. 1965), Paris, SER, 1967 (ses conclusions sont discutées par Hervé LEUWERS, Robespierre, Paris, Fayard, 2014, p. 137). Selon le conventionnel Marc-Antoine BAUDOT, Notes historiques sur la Convention nationale, le Directoire, l'Empire et l'exil des votants, Paris, Jouaust, 1893, p. 40 : « [Simon Duplay] écrivait sous la dictée de Robespierre, et au besoin lui servait de secrétaire ».

(15) $\mathrm{AN}, \mathrm{F} / 7 / 4436(1)$

(16) Arne ORDING, Le Bureau de police, op. cit., en particulier, p. 37 et sq.

(17) Lettre de Simon Duplay au Comité de Salut public du 7 fructidor an II. AN, F/7/4694. 
Après un certain nombre de recherches improductives sur les cartons du Bureau de Police (AN, F/7/4435-4438), nous nous sommes tournés vers les dossiers criminels de la Police générale qui, grâce au fondamental travail de classement de Caron et Clémencet de $1948^{18}$, sont facilement et rapidement accessibles. En visionnant les dossiers relatifs à la famille Duplay (AN, F/7/4694), deux documents nous ont semblé immédiatement très importants : il s'agit des lettres écrites par Simon Duplay pour solliciter sa libération et celle d'Éléonore et de Jacques Maurice Duplay, les enfants du menuisier Maurice. Cette fois, la graphie paraît correspondre parfaitement à celle du « dossier $2 »$. En l'occurrence, il nous semble particulièrement important de signaler que dans une lettre de Duplay, ainsi qu'au verso du « dossier 2 », l'auteur, à la fin de la feuille, décide de compléter les mots en insérant juste au-dessus les lettres qu'il n'arrive pas à disposer au long de la ligne. La comparaison entre la signature de Simon, en F/7/4694, et celle contenue dans les documents de l'interrogatoire de Simon sur ses rapports avec Robespierre (AN, W//79), dissipe toute incertitude. Le document intitulé «Le Bonheur, émané de la Liberté » a été physiquement rédigé par Simon Duplay qui, vraisemblablement, a d'abord recopié ce manuscrit, puis, suivant les indications de l'auteur, a ensuite apporté les corrections nécessaires ainsi que les ratures que l'on peut reconnaître dans le texte « final» parvenu jusqu'à nous ${ }^{19}$.

Bien que nous soyons arrivés à Simon Duplay en suivant la piste des secrétaires de Robespierre, il n'est pas certain que Simon n'ait été que le copiste de l'Incorruptible. En effet, malgré la possibilité que Simon ait travaillé sous la dictée de Maximilien - le problème posé par la partie du texte où l'auteur utilise la locution «mes enfans » reste encore une question ouverte :

« [...] je voyois autour de moi $\mid$ croitre mes enfans, mais je me disais : peut-être un jour ils | seront les victimes de [ła vie] l'opulence et du crime. peut être | un jour ils seront jettés dans ces gouffres [?] où gemissent tant $\mid$ de malheureux, parcequ'ils auront aimé la vertu et leur | patrie. »

Bien que Robespierre et Simon Duplay n'aient pas d'enfants en 1794, il est possible d'affirmer que, d'un côté - comme l'a récemment

(18) Index ms., sur fiches, par Pierre Caron et Suzanne Clémencet, 1948. 29 boîtes de fiches (Caran, 9 vol. xérographiés). Pierre Caron et Suzanne Clémencet ont catalogué les cartons de F/7/4577 à $F / 7 / 4775 / 53$.

(19) Nous soulignons que le «dossier 2 » ne contient pas un texte définitif mais un travail encore in itinere. 
mis en évidence Annie Geffroy - l'usage de cette formule peut être une figure rhétorique, utilisée par Robespierre pour rendre le discours davantage pathétique, ou encore faire référence à ses frères ou aux enfants du couple Duplay $^{20}$; d'un autre côté, cependant, les mots mentionnés nous semblent précisément celles d'un père inquiet pour le sort de ses enfants...

Suivant cette dernière hypothèse, il n'est pas exclu que Robespierre ne soit pas l'auteur du « Bonheur émané de la Liberté ». En effet, en plus du passage que nous venons de citer, il nous interpelle aussi sur le fait qu'aucun des historiens ayant eu accès aux documents Le Bas au XIX ${ }^{\mathrm{e}}$ siècle, n'ait signalé ce manuscrit ; en particulier, il n'est pas cité par Ernest Hamel, dont l'œuvre en trois tomes cite un nombre important de documents ${ }^{21}$. D'ailleurs, il est reconnu que les documents de Robespierre saisis au 9 Thermidor sont d'une composition hétérogène ; on y retrouve, en effet, des notes, des lettres, des discours manuscrits ainsi que des missives ou des rapports qui lui sont adressés, des arrêtés du Comité de salut public, des notes d'autres fonctionnaires, etc ${ }^{22}$. Les documents conservés par les Le Bas semblent avoir la même composition, comme le montre aussi le cas $\mathrm{du}$ « Discours sur les finances $»^{23}$ qui, selon Hervé Leuwers et moi-même, ne peut aucunement être attribué à Robespierre.

En l'état actuel de nos recherches, nous ne pouvons finalement que laisser ouverte la question de l'auteur du document, ne pouvant ni confirmer ni rejeter la possibilité que «Le Bonheur émané de la Liberté » soit un texte robespierriste.

En ce qui concerne le destinataire de cette « lettre », nous n' avons aucun document susceptible de l'identifier avec certitude. Par conséquent,

(20) Nous soulignons quand même comment Élisabeth Duplay-Le Bas affirme - dans les mémoires publiées par Stéfane-Pol (Le Conventionnel Le Bas, op. cit., p. 102 et sq.) - que le rapport qui liait Maximilien au couple Duplay était similaire au rapport enfant-parents, et que celui qui liait Robespierre aux enfants des Duplay était comparable au rapport entre un frère aîné et ses frères et sœurs plus jeunes : « [Robespierre] avait un profond respect pour mon père et ma mère ; aussi le regardaient-ils comme un fils, et nous comme un frère ».

(21) À ce propos il y a trois scénarios possibles : 1) Hamel n'a pas considéré la « lettre » assez importante pour la signaler ; 2) il n'a pas pu la voir, parce que la lettre n'était pas parmi les autres documents conservés initialement par Élisabeth Duplay-Le Bas. Dans ce cas il faudrait comprendre quand et par qui la « lettre » aurait été ajoutée au dossier sur Robespierre en possession de la famille Le Bas ; 3) il est possible que Hamel ait écarté ce document, en le considérant difficile à attribuer.

(22) Cf. AN, F/7/4436 (1). Dans le même carton il y a aussi l'Inventaire des pièces originales remises par le citoyen Courtois au nom de la Commission chargée de vérifier les papiers trouvés chés [sic] Robespierre et autres conspirateurs, à la Commission des 21 le neuf pluviose an 3 de la Rep[publi]que. Cf. aussi Papiers inédits trouvés chez Robespierre, Saint-Just, Payan, etc., 3 tomes, Paris, Baudouin frères, 1828.

(23) Cf. AN, 683 AP/1 (dossier 1). 
nous nous limiterons ici à de simples remarques. Certainement, l'hypothèse suggérée par Annie Geffroy, selon laquelle il pourrait s'agir du brouillon d'une lettre de Maximilien adressée à son frère Augustin paraît intéressante. La lecture des premiers mots raturés du texte pemettraient-ils de le confirmer ? Quoi qu'il en soit, mentionnons la suggestive hypothèse de Cesare Vetter ${ }^{24}$, selon laquelle l'auteur pourrait avoir pour interlocuteur le Rousseau des Rêveries du promeneur solitaire ${ }^{25}$, rédigées pendant ses dernières années de vie à Ermenonville. Néanmoins, il nous semble qu'à plusieurs reprises «Le Bonheur émané de la Liberté », tout en inscrivant dans l'imaginaire propre de la pensée de Rousseau, s'apparente plutôt aux dernières pages de l'Émile, dans lequel le philosophe fait dialoguer les raisons de la vie solitaire avec celles de la vie en société. En ce sens, le « dossier 2 » ressemble plus à une lettre écrite par l'instituteur à Émile qu'à un dialogue à distance entretenu avec le philosophe genevois, comme il est possible de le noter dans les passages suivants avec lesquels nous terminons notre note :

Rousseau : [Émile] Que m'importe tous vos beaux emplois et toutes les sottes opinions des hommes? Je ne connais point d'autre gloire que d'être bienfaisant et juste ; je ne connais point d'autre bonheur que de vivre indépendant avec ce qu' on aime [...] Je ne veux pour tout bien qu'une petite métairie dans quelque coin du monde. Je mettrai toute mon avarice à la faire valoir, et je vivrai sans inquiétude. Sophie et mon champ, et je serai riche.

« Dossier 2 » : Tu croyois, cher ami, qu'il suffisait à l'homme, pour être heureux, | de vivre solitaire, [eccupé seul] dans le sein de la nature, mépriser ces | vains noms, enfans de l'orgueil et de la tyrannie, et fouler aux pieds | le vain éclat de l'or. Qu'il est heureux, me disais tu, celui qui, | éloigné du fracas turbulent de la ville, goutant une paix éternelle $\mid$ dans sa cabanne, adorant une epouse qui fait son bonheur, voit $\mid$ autour de lui ses enfans, lui tendre les mains et l'appeller leur $\mid$ père.

(24) Cf. Cesare VetTER, Marco MARIN, La felicità è un'idea nuova in Europa. Contributo al lessico della rivoluzione francese, t. 2, Trieste, EUT, 2013, p. 28, n. 1.

(25) Jean-Jacques RouSSEAU, Les rêveries du promeneur solitaire, dans Euvres complètes, édition publiée sous la direction de Bernard GAGNEBIN et Marcel RAYMOND, avec la collaboration de François Bouchardy, Jean-Daniel CANDAUX, Robert DERARTHÉ, Jean FABRE, Jean STAROBINSKI et Sven STELling-Michaud, 5 vol., Paris, 1959-1995, vol. 1, p. 993-1097. Version en-ligne, URL : http://fr.wikisource.org/wiki/Collection_compl\%C3\%A8te_des_\%C5\%93uvres_de_J._J._ Rousseau. 
Rousseau : [Instituteur d'Émile] Oui, mon ami, c'est assez pour le bonheur du sage d'une femme et d'un champ qui soient à lui ; mais ces trésors, bien que modestes, ne sont pas si communs que vous pensez. [...] Un champ qui soit à vous, cher Émile ! et dans quel lieu le choisirez-vous ? [...] Avant de choisir cette heureuse terre, assurez-vous bien d'y trouver la paix que vous cherchez; gardez qu'un gouvernement violent, qu'une religion persécutante, que des mœurs perverses ne vous y viennent troubler. Mettez-vous à l'abri des impôts sans mesure qui dévoreraient le fruit de vos peines, des procès sans fin qui consumeraient votre fonds. Faites en sorte qu'en vivant justement vous n'ayez point à faire votre cour à des intendants, à leurs substituts, à des juges, à des prêtres, à de puissants voisins, à des fripons de toute espèce, toujours prêts à vous tourmenter si vous les négligez ${ }^{26}$.

«Dossier 2 »: Tu te croyois heureux, et tu ne goutais que l'ombre du | bonheur. À côté de ta chaumiere était un laboureur languissant | sous le poids des impots ; ici c'était un homme vertueux victime $\mid$ du despotisme et du crime. Tu ausais [sic] te dire heureux, lorsque $\mid$ tes semblables étaient abreuvés d'amertumes; tu ausais te dire | heureux lorsque ta patrie gemissait sous la tyrannie d'un $\mid$ despote et de ses courtisans. Insensé tu te croyois donc seul | sur la terre ; et parceque leur fureur n'avoit point encore | pénétré dans ton azile, ton ame se fermait aux cris de la vertu | opprimée. [...] un despote insolent et barbare, dictant, lui seul, des lois à un grand peuple qu'il $[\mathrm{au}]<\mathrm{o}>$ sait appeller ses sujets ; des prêtres ambitieux [qui se faisant avec échelle, des] < imolant des milliers de victimes $>$ au nom d'un être bienfaisant, qu'ils rendaient féroce et barbare, la justice exilée, le crime toujours plus fort que la vertu.

Marco MARIN

Chercheur à l'Université de Trieste marcomarin77@yahoo.it

(26) Jean-Jacques RousSEAU, Émile ou de l'éducation (1762), dans Euvres complètes, op. cit., vol. IV, p. 239-867, 1. V, p. 856-857.

Édition en ligne, URL : https://fr.wikisource.org/wiki/\%C3\%89mile,_ou_De_1\%E2\%80\%99\%C3\% A9ducation. 